\title{
The Influence of Tthe Grinding Grains Shape and Orientation on Performance of Coated Abrasive Tools
}

\author{
Dmitry Shatko ${ }^{1, *}$ Vladimir Lyukshin ${ }^{1}$ and Pavel Strelnikov ${ }^{1}$ \\ ${ }^{1}$ T.F. Gorbachev Kuzbass State Technical University, 28 Vesennyaya, Kemerovo, 650000, Russia
}

\begin{abstract}
The issues of the influence of the orientation and shape of grinding grains of coated abrasive tools on their basic performance characteristics are considered in the article. The advantages of machining with flexible grinding tools are listed. The features of the abrasive grains contained in tools on a flexible basis are presented. The low efficiency of using standard tools is justified. A method is described for increasing the efficiency of the grinding process with tools on a flexible basis due to an integrated approach to the design of new tool designs. It is proposed to use abrasive grains of a given shape and oriented in a certain way relative to the surface of the tool base. The main stages of work related to the development of new tool designs and studies of their operational characteristics are presented. Experimental data of the dependencies of cutting capability, wear, temperature in the cutting zone, power spent on cutting, and the cut surface quality on the shape and the orientation angle of grinding grains are presented. Practical recommendations for the application of tools containing in their structure certain varieties of grinding grains are proposed.
\end{abstract}

\section{Introduction}

Grinding of parts with coated abrasive tools, such as sanding belts and flap wheels, has found wide distribution in many industries. This type of grinding has a number of advantages, such as the grinding surface elasticity, the low power loads, and the low heat density of the process.

A distinctive feature of coated abrasive tools is the working conditions of grains, which, as a rule, is levelled in height during operation and evenly distribute the load among them, which has a positive effect on the grinding process [1-4].

Despite the large number of advantages presented above, there is a relatively low efficiency in the use of the potential capacities of coated abrasive tools, as well as of any other abrasive tools. In many ways, this result is predetermined by the use of standard abrasive in the manufacture of tools, when the shape and orientation parameters of grains relative to the substrate are not taken into account [5-11].

\footnotetext{
* Corresponding author: shdb.tm@kuzstu.ru
} 
This approach to the design and manufacture of grinding tools leads to the fact that most of abrasive grains do not participate properly in the cutting process [12-15].

In this paper, it is proposed to improve the efficiency of the grinding with coated abrasive tools due to an integrated approach to the development of new designs based on the use of abrasive grains of a given shape and oriented in a certain way relative to the surface of the substrate. This design activity has great relevance and practical significance, since fuller use of the potential of each individual grain in the structure of the tool allows achieving higher grinding performance.

The purpose of the work is to improve the grinding process through the use of new designs of coated abrasive tools.

The tasks of the work include a comprehensive study of issues related to the design and development of new designs of coated abrasive tools, as well as full research of their performance.

\section{Theoretical and experimental research}

The theoretical studies are based on mathematical calculations, programming and computer simulation with the use of computer technology. The experimental part of the work was carried out in laboratory and in a production environment.

During the period of work on this topic, a step solution of the following tasks was carried out:

1. The concept of creating new designs of flap wheels and sanding belts was developed, which is based on the use of abrasive grains pre-classified according to their shape, which are in a certain way oriented relative to the substrate surface. As an example, fig. 1 shows the surface of the coated abrasive tool, containing in its structure lamellar-shaped abrasive grains, which are oriented perpendicular to the surface of the substrate.

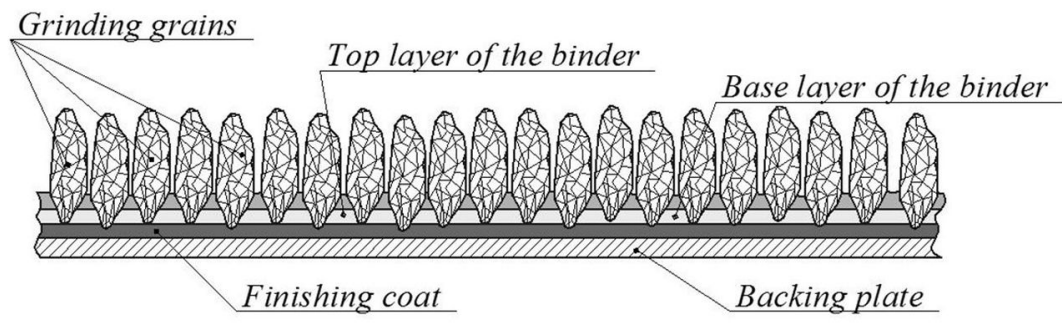

Fig. 1. Coated abrasive tool surface area.

2. Laboratory equipment for the manufacture of new tools (a separator for the separation of grinding grains on the basis of their shape, a compact electrostatic abrasive application line and a drying heat chamber) was designed and constructed.

3. A progressive method of quantifying the shape of grains using the shape factor and computer was used.

4. Statistical models were developed that reflect the influence of the shape and the orientation of grains on coated abrasive tool performance.

5. Original methods were developed for the manufacture of fundamentally new designs of flap wheels and sanding belts, for which a number of patents for inventions of the Russian Federation were obtained (No. 2245240, No. 2240224, No. 2345879, No. 2250817).

6. An experimental batch of new flap wheels and sanding belts was manufactured using grains with a controlled shape and a given orientation.

7. Some many-sided researches were conducted aimed at studying the influence of the shape and the orientation of abrasive grains of tools on their performance. 
8. Practical recommendations on the use of flap wheels and sanding belts of new designs are formulated.

\section{Results and discussion}

Some experimental data demonstrating the importance of factors such as the shape and the orientation of abrasive grains in the structure of sanding belts are presented below.

Fig. 2 shows the dependence of the cutting capacity of the grinding belt on the shape factor and the grain orientation. In this diagram, as well as in the dependencies given below, the results of S235 (HB111) steel surface grinding are presented. In addition to this steel grade, studies were also carried out for grades $100 \mathrm{Cr} 6$ and $\mathrm{X} 20 \mathrm{Cr} 13$, which are not presented in this work.

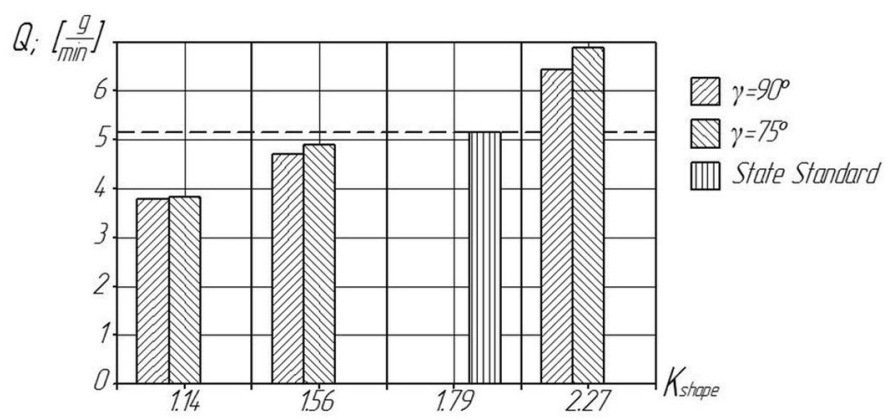

Fig. 2. Influences of the shape $\left(K_{\text {shape }}\right)$ and orientation $(\gamma)$ of grinding grains on the cutting capacity of sanding belts $(Q)$.

From the diagram it can seen that:

- the cutting capacity of grinding belts with the orientation angle $\gamma=75^{\circ}$ is higher throughout the experiment;

- the cutting capacity of grinding belts with isometric-shaped grains $\left(K_{\text {shape }} \approx 1.14\right)$ is the lowest, and for belts with lamellar-shaped grains $\left(K_{\text {shape }} \approx 2.27\right)$ oriented at an angle $\gamma=75^{\circ}$ is the highest;

- the cutting capacity of experimental belts with lamellar-shaped grains $\left(K_{\text {shape }} \approx 2.27\right)$ is higher than the cutting capacity of a standard belt (manufactured according to GOST State Standard) by $30 \div 40 \%$, and with isometric-shaped grains $\left(K_{\text {shape }} \approx 1.14\right)$ is lower by $25 \div 30 \%$. The cutting capacity of belts with an intermediate grain shape $\left(K_{\text {shape }} \approx 1.56\right)$ is almost the same than that of a standard belt.

The models obtained as a result of statistical processing and their correlation factors are presented in Table 1 .

Table 1. Statistical models.

\begin{tabular}{|c|c|}
\hline Dependence & Model \\
\hline Linear & $Q=7.0036+2.5411 \cdot K_{\text {shape }}-0.0139 \cdot \gamma-1.6573 \cdot t$ \\
& Correlation factor $(R)-0.9594$ \\
\hline \multirow{2}{*}{ Logarithmic } & $Q=12.2577+4.1799 \cdot \ln K_{\text {shape }}-1.1408 \cdot \ln \gamma-4.2561 \cdot \ln t$ \\
& Correlation factor $(R)-0.9827$ \\
\hline \multirow{2}{*}{ Exponential } & $Q=\exp ^{(2.0262+0.463 \cdot K s h a p e-0.0033 \cdot \gamma-0.3443 \cdot t)}$ \\
& Correlation factor $(R)-0.9944$ \\
\hline
\end{tabular}

Table 1 proves that the experimental data obtained are described with sufficient accuracy by an exponential dependence. 
From the analysis of models it can be seen that the grinding grain shape has the greatest influence on the cutting capacity. This is explained by the fact that with an increase in the factor Kshape, the shape of the grains changes from isometric to lamellar. Lamellar-shaped grains are deeper embedded in the material being cut, leaving deeper marks on the surface of the part, and cutting off chips of a larger cross-section, which generally increases the cutting capacity, all other things being equal. Thus, increasing the parameter Kshape, i.e. using lamellar-shaped grains it is possible to achieve an increase in cutting capacity. From Table 1 it follows that the cutting capacity decreases over time. The influence of the angle of orientation of grinding grains is least pronounced. However, it can be seen that as the angle of inclination of grains $(\gamma)$ increases, the cutting ability falls.

Fig. 3 shows the dependence of the grinding belt wear on the shape factor and the orientation of grains.

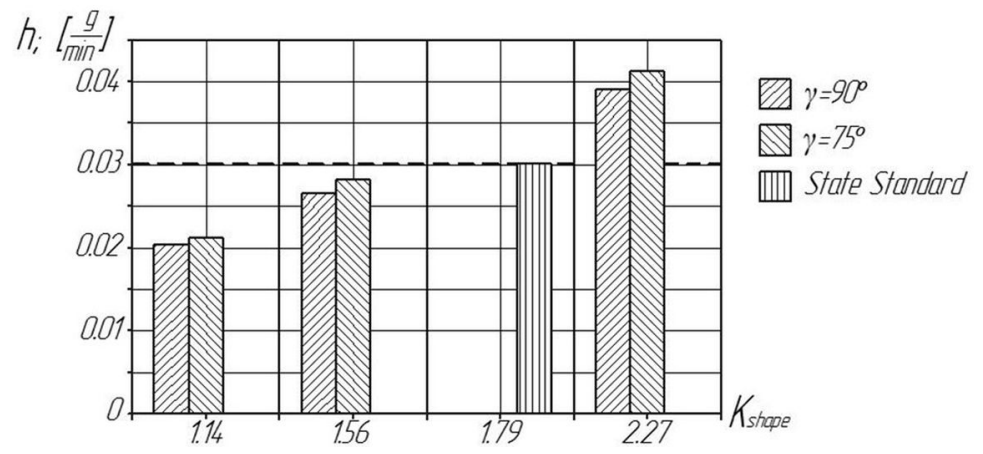

Fig. 3. Influences of the shape $\left(K_{\text {shape }}\right)$ and orientation $(\gamma)$ of grinding grains on the wear of sanding belts $(h)$.

From the diagram it can be seen that:

- wear of sanding belts with the orientation angle $\gamma=75^{\circ}$ is higher throughout the experiment;

- wear of sanding belts with isometric-shaped grains $\left(K_{\text {shape }} \approx 1.14\right)$ is the lowest, and that of belts with lamellar-shaped grains $\left(K_{\text {shape }} \approx 2.27\right)$ oriented at an angle $\gamma=75^{\circ}$ is the greatest; - wear of experimental grinding belts with lamellar-shaped grains $\left(K_{\text {shape }} \approx 2.27\right)$ is higher than that of a standard belt (manufactured according to GOST - State Standard) by $30 \div 40 \%$, and with of isometric-shaped grains $\left(K_{\text {shape }} \approx 1.14\right)$ is lower by $30 \div 35 \%$. Wear of belts with an intermediate shape grains $\left(K_{\text {shape }} \approx 1.56\right)$ is almost the same than that of a standard belt.

The models obtained as a result of statistical processing and their correlation factors are presented in Table 2.

Table 2. Statistical models.

\begin{tabular}{|c|c|}
\hline Dependence & Model \\
\hline Linear & $h=0.0569+0.0174 \cdot K_{\text {shape }}-0.0001 \cdot \gamma-0.016 \cdot t$ \\
& Correlation factor $(R)-0.8494$ \\
\hline \multirow{2}{*}{ Logarithmic } & $h=0.0953+0.0285 \cdot \ln K_{\text {shape }}-0.0084 \cdot \ln \gamma-0.0441 \cdot \ln t$ \\
& Correlation factor $(R)-0.9311$ \\
\hline Exponential & $h=\exp ^{(-2,3713+0.5388 \cdot K \text { Khape }-0.0038 \cdot \gamma-0.7904 \cdot t)}$ \\
& Correlation factor $(R)-0.9857$ \\
\hline
\end{tabular}

When comparing the calculated correlation factors $(R)$, it can be seen that the obtained experimental data are described with sufficient accuracy by an exponential dependence. 
From the analysis of the models it can be seen that the shape of grinding grain has the greatest effect on wear. Wear decreases over time. This is explained by the fact that at the initial stage of work, weakly bonded and protruding grains are removed from the working surface of the sanding belt. At the next stage, the grinding grains, having sharp edges, selfsharpen, and as they blunt, they begin to wear true. With an increase in Kshape, wear increases. This is due to the fact that lamellar-shaped grinding grains have low strength and are more prone to self-sharpening, and the isometric-shaped grains, possessing high strength, wear forming the worn place.

Fig. 4 shows the temperature in the cutting zone as a function of the shape and the orientation of grains.

From the diagram you it can be seen that:

- the temperature in the cutting zone of grinding belts with isometric-shaped grains $\left(K_{\text {shape }} \approx 1.14\right)$ is the lowest, and for belts with lamellar-shaped grains $\left(K_{\text {shape }} \approx 2.27\right)$ is the highest;

- the temperature in the cutting zone of grinding belts with the orientation angle $\gamma=75^{\circ}$ is higher throughout the experiment;

- the temperature in the zone of cutting with experimental grinding belts with lamellarshaped grains $\left(K_{\text {shape }} \approx 2.27\right)$ is $11 \div 15 \%$ higher than the temperature in the zone of cutting with a standard tape (manufactured according to GOST - State Standard), and with isometric-shaped grains $\left(K_{\text {shape }} \approx 1.14\right)$ is $10 \div 13 \%$ less. The temperature in the cutting zone of belts with an intermediate grains $\left(K_{\text {shape }} \approx 1.56\right)$ do not differ much from that of a standard belt.

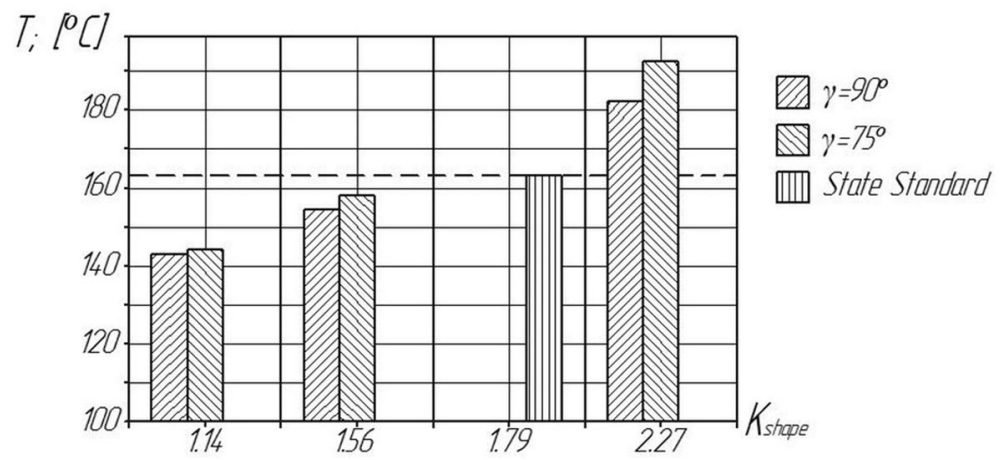

Fig. 4. Influences of the shape $\left(K_{\text {shape }}\right)$ and orientation $(\gamma)$ of grinding grains on the temperature in the cutting zone $(T)$.

The models, obtained as a result of statistical processing, and their correlation factor are presented in Table 3.

Table 3. Statistical models.

\begin{tabular}{|c|c|}
\hline Dependence & Model \\
\hline Linear & $T=137.6449+43.1419 \cdot K_{\text {shape }}-0.3333 \cdot \gamma-6.8833 \cdot t$ \\
& Correlation factor $(R)-0.8698$ \\
\hline Logarithmic & $T=260.54+71.08 \cdot \ln K_{\text {shape }}-27.42 \cdot \ln \gamma-12.26 \cdot \ln t$ \\
\hline \multirow{2}{*}{ Exponential } & Correlation factor $(R)-0.8253$ \\
\hline
\end{tabular}

From Table 3, it can be seen that the experimental data obtained are described with sufficient accuracy by an exponential dependence. 
From the analysis of the models, it follows that the shape of grinding grain has the greatest influence on the temperature in the cutting zone. With an increase in Kshape, the temperature in the cutting zone increases. This is explained by the fact that the number of grains per unit surface of the sanding belt also increases with an increase in Kshape, and, consequently, the number of heat impulses increases. The effect of the orientation angle is less pronounced. It is seen that with decreasing angle, the temperature in the cutting zone increases.

Fig. 5 shows the dependence of the power spent on cutting on the shape and the orientation of grains.

From the diagram it can be seen that:

- sanding belts with the orientation angle $\gamma=75^{\circ}$ consume more power for cutting throughout the experiment;

- the power consumption of the experimental grinding belts with lamellar-shaped grains $\left(K_{\text {shape }} \approx 2.27\right)$ is higher than power spent on cutting with a standard tape (manufactured according to GOST - State Standard) by $20 \div 30 \%$, and with isometric-shaped grains $\left(K_{\text {shape }} \approx 1.14\right)$ is lower by $15 \div 25 \%$. The power consumption of belts with intermediateshaped grains $\left(K_{\text {shape }} \approx 1.56\right)$ is almost the same than that of a standard belt.

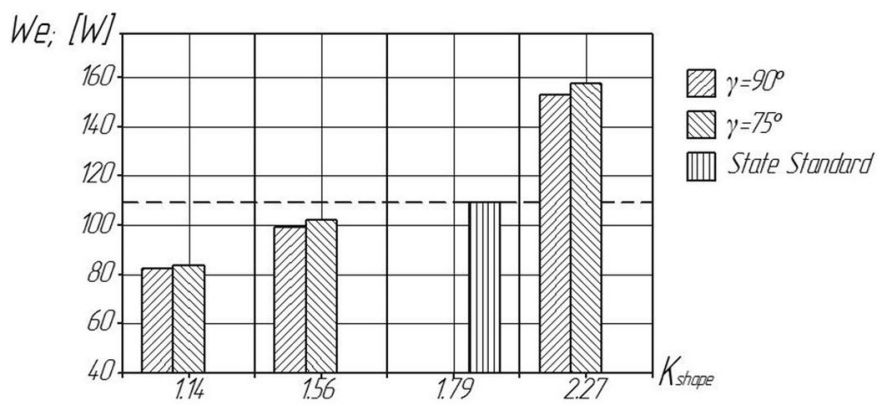

Fig. 5. Influences of the shape $\left(K_{\text {shape }}\right)$ and orientation $(\gamma)$ of grinding grains on the power spent on cutting $(\mathrm{We})$.

The models obtained as a result of statistical processing and their correlation factors are presented in Table 4.

Table 4. Statistical models.

\begin{tabular}{|c|c|}
\hline Dependence & Model \\
\hline Linear & $W e=78.0385+65.188 \cdot K_{\text {shape }}-0.1822 \cdot \gamma-19.4333 \cdot t$ \\
& Correlation factor $(R)-0.9816$ \\
\hline \multirow{2}{*}{ Logarithmic } & $W e=173.35+106.1 \cdot \ln K_{\text {shape }}-14.99 \cdot \ln \gamma-145.85 \cdot \ln t$ \\
Correlation factor $(R)-0.9496$
\end{tabular}

When comparing the calculated correlation factors $(R)$, it can be seen that the obtained experimental data are described with sufficient accuracy by a linear dependence.

From the analysis of the models it can be seen that the shape of grinding grain has the greatest impact on power consumption. This is explained by the fact that with an increase in Kshape, the shape of grinding grains changes from isometric to lamellar, and, as is already known, sanding belts with lamellar-shaped grains have a greater cutting capacity and, consequently, spend more power on cutting. From Table 4 it follows that power spent on cutting decreases over time. The influence of the angle of orientation of grinding grains is least pronounced. However, it can be seen that as the angle increases, the power consumption decreases. 
Fig. 6 shows the dependence of the cut surface quality on the shape factor and grain orientation.

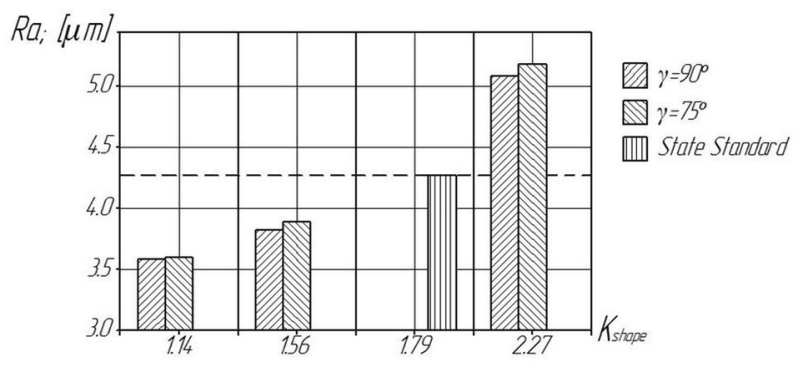

Fig. 6. Influences of the shape $\left(K_{\text {shape }}\right)$ and orientation $(\gamma)$ of grinding grains on the surface quality index $(R a)$.

From the diagram it can be seen that: - the roughness factor of the surface obtained by sanding belts with the orientation angle $\gamma=75^{\circ}$ is higher throughout the experiment;

- the roughness factor of the surface obtained by sanding belts with isometric-shaped grains $\left(K_{\text {shape }} \approx 1.14\right)$ is the lowest, and by belts with lamellar-shaped grains $\left(K_{\text {shape }} \approx 2.27\right)$ is the highest;

- the roughness factor of the surface obtained by the experimental tapes with lamellarshaped grains $\left(K_{\text {shape }} \approx 2.27\right)$ is higher than the roughness factor of the surface obtained by a standard tape (manufactured according to GOST - State Standard) by $20 \div 30 \%$, and with grains of isometric form $\left(K_{\text {shape }} \approx 1.14\right)$ is lower by $15 \div 20 \%$. The roughness factor of belts with an intermediate grain shape $\left(K_{\text {shape }} \approx 1.56\right)$ almost do not differ on the roughness factor of a standard belt.

The models obtained as a result of statistical processing and their correlation factors are presented in Table 5.

Table 5. Statistical models.

\begin{tabular}{|c|c|}
\hline Dependence & Model \\
\hline Linear & $R a=2.4505+1.3979 \cdot K_{\text {shape }}-0.0036 \cdot \gamma-0.099 \cdot t$ \\
Correlation factor $(R)-0.9742$
\end{tabular}

When comparing the calculated correlation factors $(\mathrm{R})$, it can be seen that the obtained experimental data are described by an exponential dependence with sufficient accuracy.

From the analysis of the models it is clear that the shape of grinding grain has the greatest influence on the surface finish. Moreover, with an increase in Kshape, the roughness factor increases. It results from the fact that lamellar-shaped grinding grains are sharper, and isometric-shaped ones are blunter. Thus, reducing the parameter Kshape, i.e. using grains of an isometric shape, it is possible to reduce the resulting surface roughness. The influence of the angle of orientation of grinding grains and the grinding time is the least.

\section{Conclusions}


1. The cutting capacity of belts can be significantly increased through the use of lamellarshaped grains $\left(K_{\text {shape }} \approx 2.27\right)$, oriented relative to the substrate at the angle of $\gamma=75^{\circ}$.

2. Decreasing Kshape parameter, i.e. using isometric-shaped grains it is possible to reduce wear. The degree of influence of the angle of orientation of grinding grains on wear is negligible. An increase in the angle results in less wear.

3 . The temperature in the cutting zone can be reduced through the use of sanding belts with isometric-shaped grains $\left(K_{\text {shape }} \approx 1.14\right)$.

4. Grinding belts with isometric-shaped grains $\left(K_{\text {shape }} \approx 1.14\right)$ consume less power for cutting, and belts with lamellar-shaped grains $\left(K_{\text {shape }} \approx 2.27\right)$ - the greatest.

5. The cut surface finish can be significantly improved through the use of sanding belts with isometric-shaped grains $\left(K_{\text {shape }} \approx 1.14\right)$.

\section{References}

1. H. Jamshidi, M. Gurtan, E. Budak, Mater. Proc. Techn., 273,116239 (2019)

2. V. Lyukshin, AMM, 682, 148 (2014)

3. D. Shatko V. Lyukshin, V. Bakumenko, Mater. Sci. Eng., 142, (2016)

4. N.V. Baidakova, V.A. Nazarenko, S.A. Kryukov, T.A. Baidakova, Rus. Eng. Res., 29, 509 (2009)

5. A.S. Yanyushkin, D.A. Rychkov, Proc. Eng., 206, 944 (2017)

6. V. Lyukshin, A. Barsuk, R. Fazleev, Mater. Sci. Eng., 91, (2015)

7. Y. Falkenberg, Grind. and Abr., 27 (2002)

8. S. Xu, J. Zhao, W. Zou, Diam. and Abr. Eng., 2, 69 (2007)

9. Y. Rouabhi, A. Lounis, IJCCE, 37, 241 (2018)

10. N.V. Baidakova, T.N. Orlova, Pro. Eng., 206, 194 (2017)

11. A. Yanyushkin, D. Rychkov, D. Lobanov, AMM, 682, 183 (2014)

12. S.A. Kryukov, N.V. Baidakova, P.Y. Bochkarev, LNME, 9783319956299, 1427 (2019)

13. E. Vasil'ev, A. Popov, D. Rechenko, Russ. Eng. Res. 32, 730 (2012)

14. N.V. Baidakova, T.N. Orlova, Pro. Eng., 206, 188 (2017)

15. S.A. Kryukov, N.V. Baidakova, LNME, 9783319956299, 1421 (2019) 